\title{
Effects of Healing Forest Walks for Short Breaks on Mood State, Quality of Life, and Stress Reduction of University Hospital Workers
}

\author{
Seong-Jin Han ${ }^{1}$ and Chang-Duck Koo ${ }^{2}$ * \\ ${ }^{1}$ Graduate Department of Forest Therapy, Chungbuk National University, Cheongju 28644, Korea \\ ${ }^{2}$ Department of Forestry, Chungbuk National University, Cheongju 28644, Korea
}

\begin{abstract}
This study was conducted to investigate the effects of walking in the healing forests for short breaks on mood state, quality of life and stress reduction of hospital workers. For the study, 56 employees from Chungbuk University Hospital participated in the forest walks connected to the hospital using a short break three times a week for 20 minutes each for three weeks. The Korean Version of Profile of Mood States (K-POMS) and the Korean Occupational Stress Scale (KOSS-SF) were used as validated measurement tools to evaluate changes in mood state and job stress. A 5-point Likert scale was used for assessment of quality of life among employees. The results of the questionnaire were analyzed by paired t-test. As a result, tension, anger, confusion, and fatigue in mood state categories were decreased significantly and vigor was significantly increased after forest walks. Total mood disturbance was also decreased significantly. Quality of life for hospital workers was significantly improved after forest walks. In terms of job stress, average score of stress was significantly decreased after forest walks and especially stresses from the organizational system and improper reward were significantly decreased. The results of this study proved talking a short walk in the healing forests during breaks in daily work hours has a positive effect on mood state and life quality improvements and job stress reduction in hospital workers.
\end{abstract}

Keywords: forest healing, medical services, modern society, urban forest

\section{Introduction}

The organization of general hospitals today is changing into a more multidimensional and complicated structure due to the development of medical technology and specialized structuralization, and the tasks and duties are related to human life and thus workers are facing more challenges and stress than other occupations (Kim, 2002). Moreover, the rapid development of medical technology has constantly changed the medical environment, and changes in the environment and conditions of contemporary human life including population structure and aspect of diseases are resulting in chronic illnesses, which also increases the demand for qualitative improvement of medical services (Moon, 2010). Accordingly, university hospital workers that spend most of their time at work are likely to suffer from greater

Received: August 13, 2018, Revised: September 5, 2018, Accepted: September 27, 2018

First author: Seong-Jin Han, E-mail: wisdom4242@nate.com, ORCID: 0000-0001-6933-6942

*Corresponding author: Chang-Duck Koo, E-mail: koocdm@chungbuk.ac.kr, ORCID: 0000-0001-9508-8858

2018 by the Society for People, Plants, and Environment. This is an Open Access article distributed under the terms of the Creative Commons Attribution Non-Commercial License (http://creativecommons.org/licenses/by-nc/4.0/) which permits unrestricted non-commercial use, distribution, and reproduction in any medium, provided the original work is properly cited. 
job stress than general office workers due to the organizational characteristics, such as diverse service demands of patients, work overload, and required cooperation of workers in various specialized fields of occupations (Jeon, 2013). The hospital organization is operated by mutual cooperation of workers in various lines of duty, such as doctors, nurses, medical technicians and administrative positions. Thus there are many internal stress factors and substantial amount of physical and mental labor is required. Furthermore, there are conflict factors among members of the hospital organization, and the stress intensity is known to be high at work due to the urgency of work and low substitutability (Jeong, 2002).

Job stress limits various definitions of stress only to stress at the job level, and refers to all kinds of stress related to performance of duties. The job environment and conditions interact with individuals and change their physical, physiological and psychological state with dysfunctions (Heo, 2011). Job stress of hospital workers threatens physical and mental health at the individual level and lowers organizational commitment at the organizational level, consequently decreasing job performance of the organization. Meanwhile, according to the operational definition, job stress is incurred when a job beyond one's capabilities is demanded of him or her, or when one's needs are not fulfilled by his or her surrounding environment. Therefore, when it lasts long, it causes physical health problems or mental illnesses such as insomnia and depression, causing damage to individual health (An, 2016). To resolve this issue, it is first necessary to study various factors affecting job stress and health behaviors of general hospital workers, and ultimately establish the foundation to improve the quality of life for hospital workers in charge of the quality of hospital and medical services, by discussing specific plans to minimize job stress of general hospital workers and improve lifestyles for health (Kim, 2017).

Meanwhile, various methods are presented to resolve stress, and studies are actively conducted on forest therapy as an alternative based on scientific evidence. According to Article 2 of the Forestry Culture and Recreation Act, forest therapy means immune-strengthening and health-promoting activities, which utilize a variety of elements of the forest, including scents and scenic views, and healing forest means a forest developed for forest therapy. However, many workers living in the city are isolated from forests, and it is not easy for them to regularly be close to the therapeutic forest environment in their hectic lives. Thus, by participating in forest activities using an urban forest near the hospital as a forest healing space, people can not only promote physical health through the general functions of the forest, but also enhance their mental ability to overcome stress or adversities (Kang, 2003).

Grahn and Stigsdotter (2003), who reported that taking a walk in the natural environment such as an urban park is effective for stress relief, proposed that walking as a leisure activity in an urban park is the best way to reduce stress, and argued that even a short walk reduces hormones that induce stress. De Vries et al. (2010) discovered that the benefits of urban forests include environmental improvement such as air purification, as well as stress reduction, concentration recovery and physical/physiological function recovery. This study result implies that taking a walk on a forest path can create a comprehensive synergy such as accessibility and economic feasibility (material, temporal) (Shin, 2013).

Therefore, taking a short walk in the urban park or on the forest path will have a positive effect on job stress reduction and life quality improvement of university hospital workers. However, there is almost no research on job stress and quality of life of university hospital workers with regard to forest therapy. Accordingly, this study assumes that university hospital workers taking a walk on a healing forest path during short breaks in daily life can positively change the mood state and reduce job stress, thereby improving the quality of life, and intends to examine the effects accordingly. 


\section{Research Methods}

\section{Subjects}

The subjects were 56 workers of Chunbuk National University Hospital interested in forest therapy. Their gender, age, occupation and length of service were examined as the socio-demographic background, along with their interest in forest therapy such as why they go to the forest, and preference of the five senses felt in the healing forest path, and point of visit.

\section{Study area}

The study area is 'Healing Sharing Walk' of Cheongju, Chungcheongbuk-do, which is formed for vulnerable users of transportation such as people with disabilities, elderly and infirm, and patients neglected from forest welfare benefits to more conveniently and safely enjoy the benefits of the forest. Moreover, it is connected to the 3rd floor of the main building of Chunbuk National University Hospital so that the hospital staff can enjoy leisure during work. In the research period, the temperature was minimum $12^{\circ} \mathrm{C}$ and maximum $29^{\circ} \mathrm{C}$ with the average daytime temperature of $20^{\circ} \mathrm{C}$. Classical music plays through the speakers, and the surrounding vegetation is natural forest without much diversity. The main species of trees is oak (Quercus acutissima, Quercus aliena, Quercus serrata) and it is a broadleaf forest that provides a sense of security. Most trees are taller than $20 \mathrm{~m}$, and the diameter at breast height is $26-30 \mathrm{~cm}$ on average. The slope of the deck walkway is gentle so that it is often used by not only hospital workers but also patients, guardians, caregivers and hospital visitors. The facilities include 350m deck walkway, 170m forest path, 4 deck shelters, 1 wooden bridge, 110 ground lights, and 19 bollard lights.

\section{Study plan}

The total research period was 3 weeks from September 11 to 29, 2017, and 56 participants were to take a walk on the healing forest path at least 3 times a week for 20 minutes each (Figure 1). Their mood state, quality of life and job stress were determined by taking the same survey before and after the experiment, and ex ante-ex post analysis was conducted on the results.
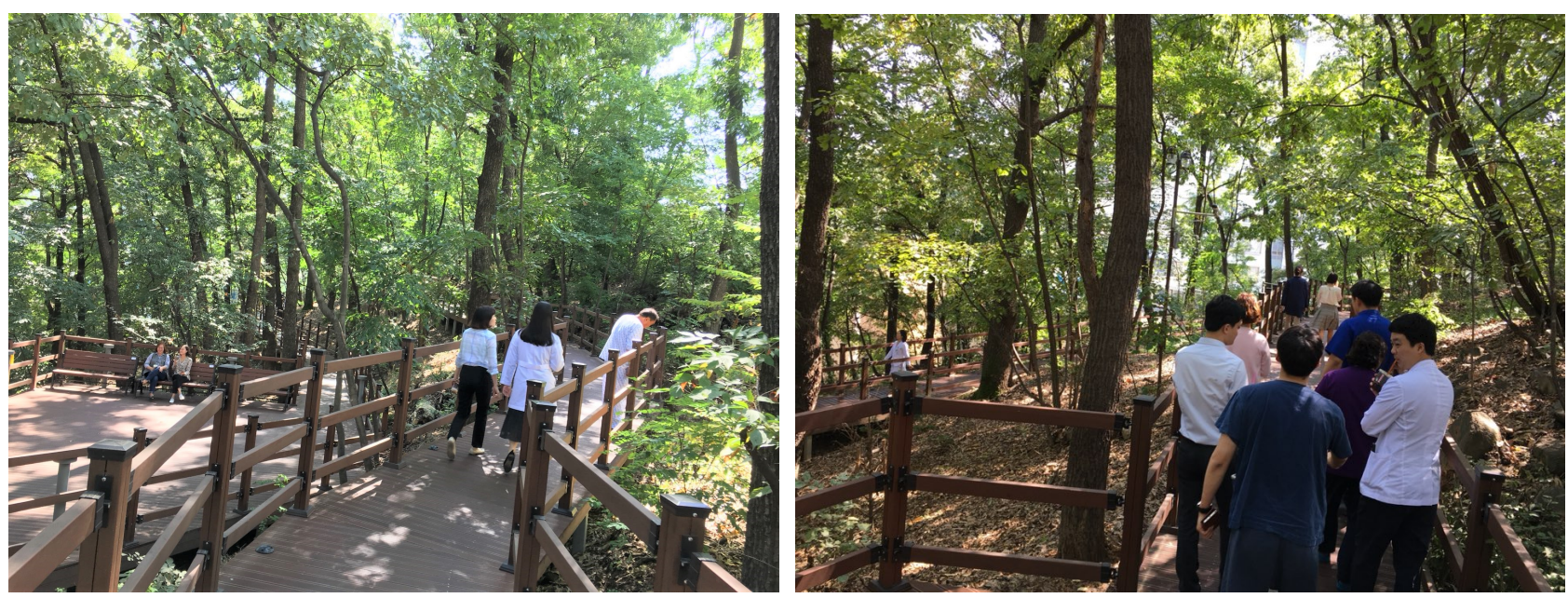

Figure 1. Pictures of hospital workers walking on Healing-Sharing Walk connected to Chungbuk University Hospital. 


\section{Measurement tools}

\section{Test of mood states (Korean Version of Profile of Mood States, K-POMS)}

Mood state is theoretically defined as a generalized emotional state permeates an individual with little stimulation perceived subjectively by the individual (McNair et al., 1971). The Korean Version of Profile of Mood States (K-POMS) is the profile of mood states (POMS) developed by McNair et al. (1971) and adapted by Kim et al. (2003), with verified reliability and validity. It was developed to quickly and conveniently apply the temporary and variable state of affect to clinical practice. This scale is classified into 6 factors: tension-anxiety (T-A), depression (D), anger and hostility (A-H), vigor $(\mathrm{V})$, confusion $(\mathrm{C})$, and fatigue (F). Total mood disturbance (TMD) is the score obtained by subtracting vigor from the sum of all 5 other items such as tension-anxiety, depression, anger-hostility, fatigue, and confusion. This study used the shortened version consisting of 30 items. Each item was rated on a 5-point Likert scale from 'Strongly disagree' $(0$ point) to 'Strongly agree' (4 points).

\section{Quality of life}

Quality of life is theoretically defined as subjective evaluation and peace perceived by each individual in the physical, mental, social and economic aspect (No, 1988). The variable to measure in the quality of life measurement is not an objective measure of life but quality of life perceived by individuals, and thus the definition of the subjective concept of quality of life was used in the study. The tool used here is the measurement tool applied by Ryoo (2013) with verified validity based on items used by Diener et al. (1985). The survey consists of 8 items, each rated on a a 5-point Likert scale from 'Strongly disagree' (1 point) to ‘Strongly agree' (5 points), with higher score indicating higher quality of life.

\section{Job stress (Korea Occupational Stress Scale-short form, KOSS-SF)}

Job stress is theoretically defined as the negative mental or physical effect perceived by members of an organization regarding their jobs (Ahn, 2003). To measure job stress, this study used the Korea Occupational Stress Scale (KOSS-SF) developed by Chang et al. (2005) through the "Standardization of Job Stress Measurement Scale for Korean. Employees." This scale consists of 24 items in 7 sub-categories such as job demands (4 items), lack of job autonomy (4 items), job instability ( 2 items), relationship conflict (3 items), organizational system (4 items), improper reward (3 items), and work culture (4 items). Each item was rated on a 4-point Likert scale from 'Strongly disagree' (1 point) to 'Strongly agree' (4 points), with higher score indicating higher job stress.

\section{Data analysis}

The collected data was analyzed using the SPSS Windows 22.0 statistical program, and a reliability analysis was conducted on the tools in 3 categories of mood state, quality of life and job stress using the same questionnaire before and after taking walks on the healing forest path. A paired t-test was conducted for mean comparison at the significance levels of $5 \%$ and $1 \%(p<.05, p<.01)$.

\section{Results and Discussion}

\section{Socio-demographic background and interest in forest therapy}

General characteristics of the subjects are as follows. Fifty three out of 56 participants were female, and most workers were women such as 23 nurses and 20 medical technicians. Twenty five subjects were aged 40-49 and 15 were aged 
30-39. Forty five subjects were married, and 51 were full-time permanent employees, taking up most of the subjects. Twenty two were college graduates and 15 were graduate school graduates. Twenty four subjects worked for at least 20 years, 17 of them received the monthly salary of 4 million KRW or less and 5 million KRW or less, and 28 had a religion. As a result of the survey on interest in forest therapy, 41 subjects went to the forest to take a break, and 15 went for health. Most of them (37 subjects) took a walk on the healing forest path during lunch hour, and 51 of them walked with their colleagues. Regarding the five senses felt at the healing forest path, 23 responded vision (landscape) and 15 responded smell (fragrance). Thirty five subjects responded that their current job stress has been relieved slightly compared to 3 weeks before.

\section{Changes in mood state before and after walking on the healing forest path}

Mood state refers to a generalized emotional state with an individual's subjective perception, and the reliability of variables of mood state before and after walking on the healing forest path showed the Cronbach's $\alpha$ of .90, and thus internal consistency is acceptable. Tension decreased from 1.70 to 1.47 , anger from 1.42 to 1.16 , fatigue from 1.81 to 1.55 , and confusion from 1.70 to 1.53 , while vigor increased from 1.84 to 2.02 , showing significant differences (Table 1). This study proved the positive effect that walking on the healing forest path during work reduces tension, confusion, anger and fatigue among the mood state (POMS) sub-categories except depression $(p<.056)$ and increases vigor, thereby showing consistent results with previous studies (Park, 2010). Depression before and after enjoying the scenic views of the forest did not show a significant difference, which is in line with the fact that depression caused by accumulation of negative emotions does not show a significant difference in the urban forest (Kim, 2012). Meanwhile, Jeon (2017) and Shin and Choi (2017) showed that just looking at forest images as an indirect experience reduced the mean of vigor, but the result was not statistically significant. This indicates that vigor does not show a significant result just by looking at forest landscape indoors, which proves that directly taking a walk in the healing forest significantly increases vigor.

\section{Changes in quality of life before and after walking on the healing forest path}

Quality of life indicates an individual's subjective satisfaction with human welfare and happiness, and the reliability of quality of life analysis before and after walking on the healing forest path showed the Cronbach's $\alpha$ of .718, and thus

Table 1. The change of mood state before and after walking on Healing-Sharing Walk near Chungbuk University Hospital

\begin{tabular}{|c|c|c|c|c|c|c|}
\hline \multirow{2}{*}{ Subordinate scope } & \multicolumn{2}{|c|}{ Before } & \multicolumn{2}{|c|}{ After } & \multirow{2}{*}{$\mathrm{t}$} & \multirow{2}{*}{$p$} \\
\hline & M & SD & M & SD & & \\
\hline Tension & 1.70 & 0.67 & 1.47 & 0.76 & -2.650 & $.010^{*}$ \\
\hline Depression & 1.22 & 0.76 & 1.05 & 0.76 & -1.995 & .056 \\
\hline Confusion & 1.70 & 0.51 & 1.53 & 0.50 & -3.245 & $.002^{* *}$ \\
\hline Anger & 1.42 & 0.70 & 1.16 & 0.65 & -3.683 & $.001^{* *}$ \\
\hline Fatigue & 1.81 & 0.72 & 1.55 & 0.74 & -3.052 & $.003^{* *}$ \\
\hline Vigor & 1.84 & 0.60 & 2.02 & 0.63 & 2.689 & $.009^{* *}$ \\
\hline $\mathrm{TMD}^{\mathrm{z}}$ & 40.23 & 14.79 & 35.82 & 15.01 & -2.936 & $.005^{* *}$ \\
\hline
\end{tabular}

Note. When determining the Mean; $0=$ strongly disagree; $4=$ strongly agree.

${ }^{\mathrm{z}} \mathrm{TMD}$ is total mood disturbance.

${ }^{*} p<.05, \stackrel{* *}{p} p<.01$ 
Table 2. The change of quality of life before and after walking on Healing-sharing walk near Chungbuk University Hospital

\begin{tabular}{|c|c|c|c|c|c|c|}
\hline \multirow{3}{*}{ Quality of Life } & \multicolumn{2}{|c|}{ Before } & \multicolumn{2}{|c|}{ After } & \multirow{2}{*}{$\mathrm{t}$} & \multirow{2}{*}{$p$} \\
\hline & M & SD & M & SD & & \\
\hline & 3.38 & 0.43 & 3.58 & 0.43 & 4.444 & $.00 * *$ \\
\hline
\end{tabular}

Note. When determining the Mean; $1=$ strongly disagree; $5=$ strongly agree.

*** $p<.01$

Table 3. The change of job stress before and after walking on Healing-sharing walk near Chungbuk University Hospital

\begin{tabular}{|c|c|c|c|c|c|c|}
\hline \multirow{2}{*}{ Subordinate scope } & \multicolumn{2}{|c|}{ Before } & \multicolumn{2}{|c|}{ After } & \multirow{2}{*}{$\mathrm{t}$} & \multirow{2}{*}{$p$} \\
\hline & M & SD & M & SD & & \\
\hline Job demands & 2.94 & 0.48 & 2.85 & 0.45 & -1.818 & .075 \\
\hline Job autonomy & 2.50 & 0.46 & 2.50 & 0.41 & 0 & 1.000 \\
\hline Relationship conflict & 2.19 & 0.43 & 2.10 & 0.35 & -1.716 & .092 \\
\hline Organizational system & 2.76 & 0.45 & 2.58 & 0.49 & -2.821 & $.007^{*}$ \\
\hline Improper reward & 2.61 & 0.47 & 2.49 & 0.45 & -2.103 & $.040^{*}$ \\
\hline Job instability & 2.36 & 0.48 & 2.25 & 0.53 & -1.244 & .219 \\
\hline Work culture & 2.39 & 0.55 & 2.27 & 0.59 & -1.588 & .118 \\
\hline All & 2.56 & 0.27 & 2.46 & 0.31 & -2.719 & $.009^{*}$ \\
\hline
\end{tabular}

Note. When determining the Mean; $1=$ strongly disagree; 4=strongly agree.

${ }^{*} p<.05$

internal consistency is acceptable. There was a highly significant difference with the significance level close to 0 , showing an increase from 3.38 before to 3.58 after, thereby increasing quality of life (Table 2). This is consistent with previous studies that workers in the city using their lunch hour to take a walk in a forest past in the ancient palace or park near their workplace and relaxing their mind and body have positive effects, which contribute to the improvement of quality of life through physical activities (Bang et al., 2016; Kim et al., 2017; Park et al., 2017; Lee and Shin, 2015). Moreover, this is also consistent with previous studies proving that Mindfulness-Based Stress Reduction (MBSR) improves mental and medical symptoms in walking meditation and improved quality of life (Kim, 2017).

\section{Changes in job stress before and after walking on the healing forest path}

Job stress indicates all kinds of stress related to the job, and the reliability of variables of job stress before and after walking on the healing forest path showed the Cronbach's $\alpha$ of .791, and thus internal consistency is acceptable. Organizational system decreased from 2.76 before to 2.58 after, and improper reward from 2.61 before to 2.49 after, showing statistical significance, but there was no statistical significance in sub-categories, with job demands decreasing from 2.94 before to 2.85 after, job autonomy from 2.50 to 2.50 , relationship conflict from 2.19 to 2.1 , job instability from 2.36 to 2.25 , and work culture from 2.39 to 2.27 . However, when tested with the mean of all items, there was a statistically significant result under $\mathrm{p}<.05$ from 2.56 before to 2.46 after (Table 3 ). This is consistent with previous studies that the forest around the office as a positive effect on work life (Sin et al., 2003; Yoo and Lee, 2015; Kim 2014), and that taking a walk or jogging in the forest increases lung capacity, controls cardiac impulse, and reduces tension (Park, 2008; Morita et al., 2007). Meanwhile, Kim (2017) showed similar results in only job demands of the nursing procession due to time 
pressure and work overload. Moreover, in the study by Lee (2015), the forest therapy program using 6 major treatments showed insignificant results only in the job instability item of mental health workers, which is due to job instability and thus showing different results from this study on capital- and labor-intensive group of university hospital workers.

\section{Conclusion}

This study is to determine the effects of walking on the healing forest path on mood state, quality of life and job stress of Chunbuk National University Hospital workers. Based on the objectives of this study, the following conclusions can be made. First, regarding effects of walking on the healing forest path on sub-categories of mood state, there was a decrease in depression but not statistically significant. Tension, anger, fatigue and confusion showed a significant decrease, and vigor showed a significant increase. Second, walking on the healing forest path also significantly improved the quality of life. Third, regarding effects of walking on the healing forest path on sub-categories of job stress, there was no statistical significance in job demands, job autonomy, relationship conflict, job instability and work culture, but there was statistically significant results in organizational system and improper reward. As examined above, walking on the healing forest path had a positive effect on mood state change, improved quality of life, and generally reduced job stress.

The limitations of this study are as follows. First, the research was conducted in the limited space of the healing forest path connected to the third floor of the main building targeting workers of only one university hospital, most of which were women, and thus the results of this study cannot be generalized. Second, the research was on limited jobs within the hospital and the research period was relatively short with only 3 weeks without verifying the persistence of the effect, which raises the need to conduct follow-up research on long-term effects. Encouraging people to take a walk during spare time in the nearby forest will help more workers in the city to enthusiastically participate in changing their life to become healthier. Thus, follow-up research should discuss more specific plans to minimize job stress of general hospital workers and improve lifestyles for health, and ultimately establish the grounds to improve the quality of life for hospital workers that are in charge of the quality of hospital and medical services.

\section{References}

Ahn, H.W. 2003. A study on job stress factors and job stress response, job satisfaction: A case study of K-Corporation. Master's thesis, Korea University, Seoul, Korea.

An, S.R. 2016. The Research on the job stress and quality of nursing service of one upper level general hospital. Master's thesis, Seoul National University, Seoul, Korea.

Bang, K.S., I.S. Lee, S.J. Kim, M.K. Song, and S.E. Park. 2016. The effects of urban forest-walking program on health promotion behavior, physical health, depression, and quality of life: A randomized controlled trial of office-workers. J. Korean Acad. Nurs. 46(1):140-148.

Chang, S.J., S.B. Koh, D.G. Kang, S.A. Kim, M.G. Kang, C.G. Lee, J.J. Chung, J.J. Cho, M. Son, C.H. Chae, J.W. Kim, J.I. Kim, H.S. Kim, S.C. Roh, J.B. Park, J.M. Woo, S.Y. Kim, J.Y. Kim, M. Ha, J.S. Park, K.Y. Rhee, and H.R.. Kim, J.O. Kong, I.A. Kim, J.S. Kim, J.H. Park, S.J. Huyun, and D.K. Son. 2005. Developing an occupational stress scale for Korean employees. Korean J. Occup. Environ. Med. 17(4):297-317.

de Vries, S., T. Claßen, S.M. Eigenheer-Hug, K. Korperla, J. Maas, R. Mitchell, and P. Schantz. 2010. Contribution of natural environments to physical activity: Theory and evidence base. In: Nilsson et al. (Eds.), Forests, trees and human health (pp. 205-243). Springer Netherlands. DOI:10.1007/978-90-481-9806-1_8

Diener, E., R. Emmonson, R. Larsen, and S. Griffin. 1985. The satisfaction with life scale. J. Pers. Assess. 49(1):7-15. DOI:10.1207/s15327752jpa4901_13

Grahn, P. and U.A. Stigsdotter. 2003. Landscape planning and stress. Urban For. Urban Green. 2(1):1-18. 


\section{DOI:10.1078/1618-8667-00019}

Heo, M.Y. 2011. A Thesis on nurses work-related stress, job satisfaction, character types and intention of job change. Master's thesis, Yeungnam University, Gyeongsan, Korea.

Jeon, J.Y. 2017. The Effects of direct and indirect forest experience on human psychology and physiology. Master's thesis, Chungbuk National University, Cheongju, Korea.

Jeon, S.H. 2013. A Study on differences in job stress among employees of the University Hospital: Focusing on nursing, health service, administrative positions. Master's thesis, Pusan University, Pusan, Korea.

Jeong, J.J. 2002. The Effect of hospital environment on employee's job stress. Korean J. Environ. Health Soc. 28(3):72-76.

Kang, H.Y. 2003. The secret of phytoncide. Seoul. Korea: YeoksaNet publisher.

Kim, E.J., S.I. Lee, D.U. Jeong, M.S. Shin, and I.Y. Yoon. 2003. Standardization and reliability and validity of the Korean edition of profile of mood states(K-POMS). Sleep Med. Psychophysiol. 10(1):39-51.

Kim, H.H. 2017. The relevance of job stress and health behavior according to the occupational group of the general hospital workers. Master's thesis, Chungnam National University, Daejeon, Korea.

Kim. J.M. 2002. Effects of job stress on health promotion behaviors among hospital employees. Master's thesis, Inje University, Gimhae, Korea.

Kim, J.Y., C.S. Shin, and J.K. Lee. 2017. The Effects of forest healing program on mental health and melatonin of the elderly in the urban forest. J. People Plants Environ. 20(1):95-106.

Kim, K.H. 2014. Analysis of the effects of leisure satisfaction in urban parks on work stress and the resilience of office workers: The mediative effect of psychological detachment. Doctoral dissertation, Sejong University, Seoul, Korea.

Kim, K.M. 2012. Study on the effects of forest healing according to types of recreational forests. Doctoral dissertation, Chungbuk National University, Cheongju, Korea.

Lee, S.H. 2015. The Influence of forest healing program on stress high risk group's job stress, depression, mood states, self-esteem, and HRV. Master's thesis, Chungbuk National University, Seoul, Korea.

Lee, Y.J. and C.S. Shin. 2015. Influence of forest healing user`s nature relatedness, emotion, arousal state on stress recovery- focused on women in their 50's-. J. Korean Soc. People Plants Environ. 18(2):123-128.

McNair, D.M., M. Lorr, and L.F. Droppleman. 1971. Manual for the profile of mood states. San Diego, USA: Educational and Industrial Testing Service.

Moon, S.J. 2010. Structural model of nurses' intentions of changing work places. Doctoral dissertation, Kyunghee University, Seoul, Korea.

Morita, E., S. Fukuda, J. Nagano, N. Hamajima, H. Yamamoto, Y. Iwai, T. Nakashima, H. Ohira, and T. Shirakawa. 2007. Psychological effects of forest environments on healthy adults: Shinrin-yoku (forest-air bathing, walking) as a possible method of stress reduction. Public health 121(1):54-63.

No, Y.J. 1988. An analysis of the Quality of life of middle-aged adults in seoul. Doctoral dissertation. Yonsei University School, Seoul, Korea.

Park, B.J. 2010. Experimental approach of therapeutic effect of forest recreation activies. Doctoral dissertation, Chungnam National University, Daejeon, Korea.

Park, O.H., J.Y. Lee, and M.H. Hong. 2017. Experience analysis on forest healing- based on the record of experience of forest healing. J. People Plants Environ. 20(4):385-394.

Park, Y.B. 2008. Modern enterprise organizational behavior. Seoul. Korea: Bumounsa.

Ryoo, S.B. 2013. A Study on the quality of life for social welfare workers: Focused on the effects of elderly life preparation and job satisfaction. Doctoral dissertation, Chonnam National University, Gwangju, Korea.

Shin, J.W. and J.H. Choi. 2017. The effects of viewing the forest landscape on physiological and psychological status in radiologists. J. People Plants Environ. 20(3):283-291.

Shin, O.S. 2013. Fundamental research on construction of forest trail as connecting with forest effect. Doctoral dissertation, Daegu Haany University, Daegu, Korea. 
Sin, W.S., J.J. Kim, W.J. Kim, R.H. Yoo, and B.D. Lee. 2003. The Influence of urban forests on job satisfaction and stress. J. Korean For. Soc. 92(1):92-99.

Yoo, M. and E.H. Lee. 2015. The Impact of modularized interior landscape on office workers psychological wellbeing. J. Korean Soc. People Plants Environ. 18(2):79-87. 